\title{
Wer sitzt dahinter? Das Vorzimmer
}

\author{
Erschienen in: WORTATLAS
}

Von: Markus Steinmayr

Bei vielen der zahlreichen Videokonferenzen der letzten Monate erscheint in einigen Programmen der Hinweis, dass der Zugang zum Raum durch den/die Moderator*in gewährt werden müsse. Das Warten, der Aufenthalt im Warteraum ist stets ein Aufenthalt im Ungewissen: Darf ich rein, darf ich nicht rein? Ich habe doch den Zugangscode! Aber habe ich inn wirklich, wenn ich so lange warten muss?

Bisweilen habe ich mich wie ein Höfling im Versailles des 18. Jahrhundert gefühlt, dessen Begehr es ist, das Zimmer zu betreten, in dem der König sich gerade aufhält. Ich habe mich wohl noch nie so viel in jenen Zwischenzonen des Wartens aufgehalten wie in den letzten Monaten. Es sind Aufenthalte in der Kontaktzone der Macht. Und Moderator*in bei ZOOM zu sein, bedeutet in diesen Zeiten nun einmal, Macht zu haben.

Ich antichambriere also buchstäblich, auch unter Corona-Bedingungen. Antichambrieren heißt ja nichts anderes als sich im Vorzimmer aufzuhalten. Das charmante Verhaltensideal, das mit dem Antichambrieren oft assoziiert wird, verschwindet gänzlich. Es gibt nichts Uncharmanteres derzeit als allein im Warteraum zu sein. Allein, kein Blick, kein Gespräch, kein Small Talk möglich. Wird endlich der Zugang zum Raum gewährt, so öffnet sich das Panorama einer Universität, das immer virtueller zu werden scheint. Das Soziale und das Topologische der Universität verschwindet aber nicht, es wechselt nur den Modus. Digitalisierte Meetings und ihre Organisation sind also mithin die Wiederkehr des Vorzimmers.

Diese Erfahrung der letzten Monate gibt uns die Möglichkeit, sich auf die Semantik, Symbolik, aber auch die Funktionalität jenes Warteraums zu besinnen, den viele als ,Vorzimmer‘ kennen oder kennengelernt haben: Einige vielleicht nur noch in der Erinnerung an die sogenannte ,alte' Universität in ihrer vermeintlichen Herrlichkeit, andere wiederum haben diese Erfahrungen noch nicht machen können, weil die Dozent*innen ihre Türen in der Präsenzuniversität immer offen halten. Ein Vorzimmer wird nicht benötigt, wir sind, wie es heißt, ,immer ansprechbar'. In Corona-Zeiten werden die Studierenden wieder ganz klassisch in die ZOOM-Räume hereingelassen. Arbeitsgruppenleiter*innen oder Lehrstuhlinhaber*innen mit Vorzimmer, in der irgendjemand über den Zugang zur Macht wacht, gibt es kaum noch. Ob das mit der 
Personalreduktion im nicht-wissenschaftlichen Personal zu tun hat oder mit dem Verschwinden des klassischen und angestaubten Professor*innenhabitus, bestimmt die Ungleichzeitigkeit der gegenwärtigen Universität.

Das korrekte Betreten von Zimmern und das damit verbundene Überschreiten von Türschwellen ist ein wesentlicher Bestand des universitären Verhaltens-Codex. Hier zeigt sich, wer dazu gehört und wer nicht. An Orten wie dem Vorzimmer lässt sich das vielleicht schon verschwundene Arcanum der Universität mit der Beobachtung von eingeschliffenen Verhaltensweisen verbinden. Das Vorzimmer ist ein Ort der Macht, weil es Zugänge zu universitären Machthaber*innen (Dekan*innen, Rektor*innen, Professoren*innen) regelt, einschränkt, inszeniert oder ermöglicht.

Ein Vorzimmer ist zunächst einmal eine Zone, in der man wartet. Walter Benjamin hat die Schwelle von der Grenze scharf geschieden: „Die Schwelle ist ganz scharf von der Grenze zu unterscheiden. Schwelle ist eine Zone. Wandel, Übergang, Fluten liegen im Worte ,schwellen' und diese Bedeutung hat die Etymologie nicht zu übersehen. " 1 In einer Zone wartet man also. Es wird sich etwas verändern, etwas kommt auf einen zu, von dem man noch nicht weiß, was und wie es sein mag. Hinter der Tür, die noch verschlossen ist, liegt das unbekannte Terrain. Die Zone ist damit ein Ort des Übergangs. Bleibt man auf der Schwelle stehen, passiert nichts, überschreitet man sie, betritt man etwas, zu dem einem normalerweise der direkte Zugang verwehrt ist.

Der Aufenthalt im Vorzimmer ist ein Aufenthalt an einem Ort, der Gelegenheiten bietet. Er ist, so gesehen, eine klassische occasio im Sinne der politischen Klugheit vorrevolutionärer Zeiten. Die okkasionelle Gelegenheit kommt nämlich nicht wieder, im nächsten Augenblick ist sie vorüber, in die Vergangenheit entwichen. Stanitzek fasst die Charakteristika der occasio-Lehre folgendermaßen zusammen: „Punktualität der Ereignisse, Irreversibilität der Zeit, das sind die Bedingungen unter denen politisches Handeln sich realisieren muß." 2 Daraus folgt für den/die politicus/politica, dass er/sie auf den Punkt genau reagieren und jederzeit wissen muss, dass die Gelegenheit unter ständiger Bedrohung steht, vorüberzugehen. Übertritt er oder sie nicht die Schwelle, übertritt er oder sie sie zu spät oder zu zögerlich, war er oder sie zu langsam. Das Vorzimmer ist damit ein Testlabor für den universitären Habitus.

Diese Laborsituation setzt natürlich den politischen Klugen/die politisch Kluge in ein Beobachtungsverhältnis zur Kommunikation in den Vorzimmern. Kommunikation ist nicht nur Gespräch. Wenn die Welt des Vorzimmers sich unter dem Aspekt einer sich möglicherweise bietenden Gelegenheit darbietet, die Welt der Universität aber gleichzeitig als rastloses Spiel von Ereignissen, Events Antragsroutinen, Sprechstunden, Korrekturen auftritt, dann muss der/die Vorzimmer-Philosoph*in aufmerksam sein und die Kommunikation auf den rechten Augenblick abtasten.

Das gilt insbesondere für die Kommunikation mit Vorzimmer-Insassen*innen. Die Vorzimmer-Bewohner*innen sind, mit Fontane gesprochen, occasionelle ,Trockenwohner'. Sie halten das Warten aus, der/die Besucher*in hingegen eher schlecht. Man weiß ja: Trotz des pünktlichen Erscheinens muss gewartet werden. Das Warten, der Aufenthalt in der Zone kann somit als Zuweisung von sozialen Positionen 
interpretiert werden: Priorisiert wird man gerade nicht, das Warten dient als Geduldsprobe. Vorzimmer-Besucher*innen neigen daher zur Überreflexion. Diese Überreflexion bestünde dann gerade darin in einer Verhaltens- und Kommunikationshemmung, die den/die Kandidat*in, der die Nähe des/der Machthaber*in genießen will, immer schon zu spät kommen lässt. Er/sie verharrt in der Beobachtung und vergisst das Handeln, sprich, das Überschreiten der Schwelle. Gelegenheiten, aus dem Vorzimmer heraus die Schwellen zur Macht zu überschreiten, sind also ganz im Sinne kontingenzbewusster Soziologie „nutzbare Zufälle“3.

An den Universitäten finden sich verschiedene Topologien des Vorzimmers. In den Fakultäten sind sie Reliquien alter Ordinarien-Herrlichkeit. Der Zugang zum Machthaber des Lehrstuhls ist nicht direkt möglich. Abgeschirmt, von Studierendenhorden verschont sitzen sie in ihren Zimmern und gießen die Pflanzen. Die Yucca-Palme, ihre Pflege, ja sogar die Kommunikation mit ihr wird zum Erholungsort von des Antragswesen Unbill.

In unserer Erzählung vom Vorzimmer stehen wir jetzt auf der Schwelle zum Ende und damit stehen wir im Vorzimmer des Präsidiums oder des Rektorats. Die Ethnologie hat für solche Szenarien des Übergangs den Begriff „Liminalität“ reserviert. Van Gennep hat in seinem 1909 erstmals erschienenen Buch „Les rites de passage“ unterschiedliche Phasen der Liminalität voneinander geschieden. Zunächst existiert die sogenannte Schwellenphase, die sich in der Mitte eines Übergangsrituals befindet. Übergangsrituale sind Symbole von Veränderungsprozessen und sozialem Statuswandel, der vor allem als räumlicher Grenzübertritt inszeniert wird. ${ }^{4}$ Der Übertritt ist also Übergang.

Ein typisches Exempel für diese gleichsam ethnologische Funktion findet sich in zahlreichen Vorzimmer-Szenen in Romanen. Mit innen werden Bildungsprozesse, die ja in westlichen Kulturen den Gestaltwandel hervorbringen, inszeniert. Die geradezu klassische Stelle findet sich in Goethes „Wilhelm Meisters Lehrjahre“. Im siebten Buch, in dem Wilhelm die Örtlichkeiten der Turmgesellschaft aufsuchen darf, befindet sich Wilhelm in einem „dunkeln und engen Behältnisse" 5 . Es ist, heißt es im Text, „finster um ihn“ und als versucht, „einen Schritt vorwärts“ zu gehen, kommt er nicht weiter: „Eine ihm nicht unbekannte Stimme rief ihm zu: tritt herein!"6

Die Macht des Turms und seiner Vorzimmer besteht in „Wilhelm Meisters Lehrjahre“ darin, die Übergangsrituale des Theaters, dem Wilhelm entsagt hat, zu wiederholen und sie als Initiation in die Turmgesellschaft wieder auftreten zu lassen. Schließlich wird Wilhelm als schriftliches Zeugnis sein Lehrbrief überreicht, in dem es heißt: „Die Kunst ist lang, das Leben kurz, das Urteil schwierig, die Gelegenheit flüchtig. " 7 Man kennt solche Sätze aus der Erinnerung an viele Situationen, in denen die Schwierigkeiten und Verzögerungen im Übergang sich im Rückblick als überschätzt erweisen und zur Demonstration der Banalität von Alltagsweisheiten geraten.

Und jede*r, der durch das Vorzimmer nicht der Turmgesellschaft, sondern des/der Professor*in, des/der Dekan*in, des/der Rektor*in oder des/der Präsident*in hindurchgegangen ist, macht einen sozialen Gestaltwandel durch. Er oder sie ist initiiert worden. Ihm oder ihr sind Lehrbriefe in unterschiedlichster Art überreicht worden: Hausarbeiten, Bachelor-Arbeiten, Dissertationen, Arbeitsverträge, Dokumentation von 
Berufungsverhandlungen, Ernennungsurkunden. Wer das Vorzimmer durchschritten hat, hat es auf seine oder ihre Art und Weise geschafft. Die Übergangsrituale, die wir anhand des Vorzimmers beobachten können, sind an der Universität also, mit Viktor Turner gesprochen, Orte komplexer sozialer Veränderungen und individuellen Gestaltwandels. ${ }^{8}$ Diese Veränderung zeigt sich besonders dann, wenn Orte wie das Vorzimmer allmählich verschwinden. Es entsteht eine Leere, die die Universität derzeit nicht zu füllen vermag.

\section{References}

1. Benjamin (1935ff./1991): Das Passagen-Werk. In: W.B.: Gesammelte Schriften Bd.I-VII. Hg. von Rolf Tiedemann, Hermann Schweppenhäuser u.a. Frankfurt/M.: Suhrkamp, hier Bd. V/1, S. 618.

2. Stanitzek, Georg (1989): Blödigkeit. Beschreibungen des Individuums im 18. Jahrhundert. Tübingen: Niemeyer, S. 52. https://doi.org/10.1515/9783110930450.

3. Luhmann, Niklas (1997): Die Gesellschaft der Gesellschaft, Frankfurt/M.: Suhrkamp, Bd.1, S. 447.

4. Van Gennep, Arnold (1909/1988/2005): Übergangsriten. Aus dem Französischen von Klaus Schomburg und Sylvia M. Schomburg-Scherff. Mit einem Nachwort von Sylvia M. Schomburg-Scherff. Frankfurt/M.: Campus.

5. Goethe, Johann Wolfgang von (1795/2006): Wilhelm Meisters Lehrjahre. In: J.W.v.G: Sämtliche Werke nach Epochen seines Schaffens [=Münchner Ausgabe], Bd. 5. Hg. v. Karl Richter et al. München: BTB, S. 495.

6. Goethe (1795/2006), ebd.

7. Goethe (1795/2006, S. 497.

8. Turner, Viktor 1974: Liminal to Liminoid in Play, Flow and Ritual. An Essay in Comparative Symbology. In: Rice University Studies, 60, 1974, H. 3, S. 53-94.

SUGGESTED CITATION: Steinmayr, Markus: Wer sitzt dahinter? Das Vorzimmer, in: KWI-BLOG, [https://blog.kulturwissenschaften.de/wer-sitzt-dahinter/], 12.04.2021

DOI: https://doi.org/10.37189/kwi-blog/20210412-0805 
Dieser Text wird via DuEPublico, dem Dokumenten- und Publikationsserver der Universität Duisburg-Essen, zur Verfügung gestellt. Die hier veröffentlichte Version der E-Publikation kann von einer eventuell ebenfalls veröffentlichten Verlagsversion abweichen.

DOI: $\quad$ 10.37189/kwi-blog/20210412-0805

URN: urn:nbn:de:hbz:464-20210412-121314-9 